\title{
Effect of Melatonin on Reversing Multidrug Resistance by Targeting Phosphatase and Tensin Homolog in Leukemia Cancer Cells
}

\author{
Vahid Rameshknia ${ }^{1}$, Monireh Movahedi1 ${ }^{1}$, Abbas Akhavan ${ }^{1}$, Maryam Majidinia ${ }^{2}$ and Bahman Yousefi ${ }^{3, *}$ \\ ${ }^{1}$ Department of Biochemistry, Faculty of Biological Science, North Tehran Branch, Islamic Azad University, Tehran, Iran \\ ${ }^{2}$ Solid Tumor Research Center, Urmia University of Medical Sciences, Urmia, Iran \\ ${ }^{3}$ Drug Applied Research Center, Tabriz University of Medical Sciences, Tabriz, Iran \\ * Corresponding author: Bahman Yousefi, Drug Applied Research Center, Tabriz University of Medical Sciences, Tabriz, Iran. Tel: \\ +989149117624; Email: yousefib@tbzmed.ac.ir
}

Received 2020 0ctober 13; Revised 2021 November 24; Accepted 2021 May 25.

\begin{abstract}
Background: Recently, melatonin has attracted massive attention due to its anticancer effect on various human malignancies. It has also been demonstrated that melatonin is useful in combating resistance against conventional chemotherapeutics.

Objectives: This study aimed to evaluate melatonin's effects on multidrug resistance (MDR) in human myelogenous leukemia cells.

Methods: Melatonin's cytotoxicity on the K562 and K562/doxorubicin (DOX) cell lines was assessed using 3-(4,5-dimethylthiazol-2-yl)2,5-diphenyltetrazolium bromide (MTT) assay. The expression and activity of P-glycoprotein (P-gp) were measured as well. The mRNA and protein expression levels of tensin homolog deleted on chromosome ten (PTEN) was assessed in cells. Eventually, apoptosis in cancer cells was measured through Annexin V/PI staining.

Results: Treatment with melatonin significantly increased the cytotoxicity of DOX in resistant K562 cells. The expression and activity of P-gp were attenuated following melatonin treatment. In addition, melatonin upregulated PTEN in K562/DOX cells. Melatonin also augmented apoptosis in combination with DOX.

Conclusion: Melatonin effectively increased the cytotoxic effects of DOX in K562/DOX cells through down-regulation of P-gp and upregulation of PTEN in resistant K562 cells.
\end{abstract}

Keywords: Doxorubicin, Leukemia, Melatonin, PTEN

\section{Background}

Despite significant recent improvements in the overall prognosis and the short-term and long-term chemotherapy outcomes in leukemia patients, multidrug resistance (MDR) development often results in leukemic relapse and death in 50\%-95\% of patients with cytopenic complications and resistant disease $(1,2)$. In leukemia, it has been demonstrated that alterations in p-glycoprotein (P-gp) expression, as an ATP-dependent transporter, play critical functions in developing MDR in tumor cells (3). P-gp decreases drug concentration within the cancer cells by extruding drugs outside the cells, which reduces the therapeutic efficacies of various chemotherapeutics (4). Despite a vast number of studies that have evaluated the effects of the combination of novel natural compounds and conventional chemotherapeutics in combating MDR, further research is needed for a better understanding of MDR by identifying associated signaling pathways and critical molecules. (5).

Phosphoinositide 3-kinase (PI3K)/Akt signal transduction, which is highly hyperactive in leukemia and increases the proliferation and metastasis of leukemia cells, has attracted more attention in this regard. The inhibition of this pathway is recently considered an essential therapeutic strategy (6). The activated PI3K converts phosphatidylinositol 4, 5- bisphosphate (PIP2) to phosphatidylinositol 3, 4, 5triphosphate (PIP3) (7,8). PIP3 activates Akt phosphorylation, which results in the induction of proliferation, metastasis, and inhibition of cell death, apoptosis, and ultimately tumor progression (9). Phosphatase and tensin homolog (PTEN), as the main negative regulator of $\mathrm{PI} 3 \mathrm{~K} / \mathrm{Akt}$ signaling, acts as a tumor suppressor in various tissues (10). It converts PIP3 to PIP2, which in turn suppresses the activity of Akt. PTEN can exert its antitumor activity in pathways either dependent or independent of PI3K/Akt signaling. However, the clear mechanism of PTEN regulation is still not completely understood (11).

Melatonin (N-acetyl-5-methoxy tryptamine) is a circulating rhythm regulator mainly secreted from the pineal gland (12). Various biological activities, such as antioxidative anti-inflammatory, and antiapoptotic features, have been explained for melatonin, which has made it a potent antitumorigenic agent (12). Several studies have shown that co-administration of certain chemotherapy drugs with melatonin increases cancer cells' sensitivity to these drugs (13). Melatonin can overcome MDR through the improvement of apoptosis and cellular senescence, and down-regulation of drug transporters (14). Therefore, it is suggested that the combination of melatonin with conventional agents could be a potential therapeutic strategy for reversing MDR for leukemia. In this regard, our 
previous study revealed that balaglitazone could reverse MDR by inducing the PPAR $\gamma /$ PTEN signaling pathway through reversed P-gp-mediated resistance in tumor cells (15). In addition, the results of a study conducted by Mashayekhi et al. revealed that the cotreatment of ciglitazone and doxorubicin (DOX) effectively induced apoptosis, reversed MDR, and more importantly, decreased the adverse side effects of DOX in leukemia tumor cells (16).

\section{Objectives}

This study amid to evaluate the function of melatonin in doxorubicin resistance of human myelogenous leukemia K562 cells. Therefore, the cellular proliferation and the activity levels expression of P-gp were assessed for investigating MDR. The PTEN expression was also evaluated in K562 and resistant cells (K562/DOX), following the combined treatment with melatonin and DOX.

\section{Methods}

\subsection{Cell culture}

DOX resistant human myelogenous leukemia K562 cell line (K562/DOX) and their parental K562 cell lines were cultured in vitro in the Roswell Park Memorial Institute 1640 (Invitrogen-Gibco) medium, enriched with $10 \%$ fetal bovine serum (FBS) and 100 $\mathrm{U} / \mathrm{mL}$ penicillin/streptomycin (Invitrogen-Gibco, USA). The K562/DOX cells were treated with $0.5 \mu \mathrm{M}$ of DOX and kept at $37^{\circ} \mathrm{C}$ under $5 \% \mathrm{CO}_{2}$.

\subsection{Determination of cell proliferation}

Initially, $2 \times 10^{4}$ cells/well of K562/DOX cells and their parental cells were plated in 96 well plates and treated with various DOX and melatonin concentrations, alone or in combination with each other, and incubated for $48 \mathrm{~h}$ to evaluate the cellular growth ability. At the indicated time point, an MTT assay was performed. For this purpose, cells were treated with a medium containing $10 \%$ MTT (5 $\mathrm{mg} / \mathrm{mL}$; Sigma, USA). After $4 \mathrm{~h}$ incubation with MTT solution, the supernatant was removed. In the next stage, $100 \mu \mathrm{L}$ dimethyl sulfoxide (DMSO) was added to dissolve the formazan product. The absorbance at $490 \mathrm{~nm}$ was measured by a micro plate-reader (State Fax 2100; Awareness Technology Inc., USA).

\section{Cell viability (\%) =}

$\frac{\text { The absorbance of each test }}{\text { Mean absorbance of controls }} \times 100$

Resistance factor (RF) $=\frac{\text { The IC50 value of resistant cells }}{\text { The IC50 value of parental cells }}$

\subsection{Isolation of total RNA and RT-PCR}

Total RNA of all groups was extracted using AccuZolTM reagent (Bioneer; Daedeok-gu, Daejeon,
Korea). Absorbance measurement at $260 \mathrm{~nm}$ by UV spectrophotometer was used to quantify extracted RNA. Total RNA was then reverse-transcribed using Moloney Murine Leukemia Virus (MMLV) reverse transcriptase (Promega, USA). Following total cDNA synthesis, 40 cycles of quantitative real-time polymerase chain reaction (qRT-PCR) were applied to measure the mRNA levels of $\beta$-actin, PTEN, and Pgp using SYBR Premix Ex Taq (TaKaRa Bio, Otsu, Japan) and specific primers via the Rotor-Gene ${ }^{\mathrm{TM}}$ 6000 system (Corbett Life Science, Mortlake, Australia). The target gene expression was normalized to that of $\beta$-actin, as described previously.

\subsection{Western blotting}

RIPA cell lysis buffer (Thermo Fisher Scientific) was used for cellular protein extraction. Proteins were separated on $10 \%$ sodium dodecyl sulfatepolyacrylamide gel electrophoresis (SDS-PAGE) following the measurement of total protein concentration in all groups' lysate via Bradford reagent. After transferring gels onto polyvinylidene difluoride (PVDF) membrane, 5\% skimmed milk in TBST $(0.1 \%$ Tris-buffered saline Tween 20$)$ was used to block membranes. The membrane was washed with TBST, followed by incubation in the primary and secondary antibodies. A chemiluminescence system (ECL) was used to reveal protein bands. Antibodies against P-gp, PTEN, and $\beta$-actin were provided from Abcam (Cambridge, UK).

\subsection{Rho123 cellular uptake assay}

Rho123 cellular uptake was used to evaluate MDR in resistant cells and their parental cells. After treating cells with $5 \mu \mathrm{M}$ Rho 123 (Sigma USA) and melatonin for $30,60,90$, and $120 \mathrm{~min}$ at $37^{\circ} \mathrm{C}$, and using verapamil as a positive control, the intracellular mean fluorescence intensity (MFI) was measured using an FP-6200 fluorometer (JASCO Inc. , Tokyo, Japan).

\subsection{Apoptosis assay}

Cells were treated with DOX $(0.47 \mu \mathrm{M})$ alone and in combination with melatonin $(300 \mu \mathrm{M})$. The cells were resuspended in $500 \mu \mathrm{l}$ of binding buffer and then $5 \mu \mathrm{l}$ annexin V-FITC and $5 \mu$ l propidium iodide (EXBIO Diagnostics, Czech Republic) after $48 \mathrm{~h}$ incubation and twice washing with the cold PBS. Eventually, the cells were assessed by a flow cytometer (FACSCalibur; BD Biosciences; San Jose, CA, USA) after they were kept in a dark place at room temperature for $15 \mathrm{~min}$.

\subsection{Statistical analysis}

GraphPad Prism 6.01 software was used for statistical analyses. Data were obtained as a mean \pm SD (standard deviation). Analysis of variance (ANOVA) and then Bonferroni's and Sidak's tests were used to determine the significant differences of groups. A p-value less than $0.05 \quad(\mathrm{P}<0.05)$ was 
considered statistically significant.

\section{Results}

\subsection{Melatonin decreased the viability of K562/DOX} and reversed $M D R$

An MTT assay was performed to evaluate the role of melatonin in increasing the cytotoxic effects of DOX on K562/DOX cell lines, which reverses MDR as a result. DOX inhibited both cell lines' proliferation rate in a dose-dependent manner (Figure 1A). In comparison to controls, it was found that K562 /DOX cells showed 8.29 fold resistance to DOX since the measured IC50 value of DOX in K562 /DOX and K562 cells was 3.40 and $0.41 \mu \mathrm{M}$, respectively. Afterward, the effects of melatonin on DOX cytotoxicity against
K562 and K562 /DOX cells were examined by adding $300 \mu \mathrm{M}$ melatonin to these cells. In K562 cell lines, it was observed that the addition of melatonin's to a culture medium containing DOX inhibited cellular viability, compared to DOX treatment alone. However, it was less effective compared to the effects of the combination of melatonin and DOX on K562 /DOX cells (Figure 1B). In these resistant cells, the combination of melatonin and DOX resulted in a more potent increase in DOX cytotoxicity and shift to the left of viability profile, which led to the significant inhibition of K562/DOX cells proliferation (Figure 1C). Therefore, melatonin effectively increased the cytotoxic function of DOX and overcame MDR in DOX-resistant cells.
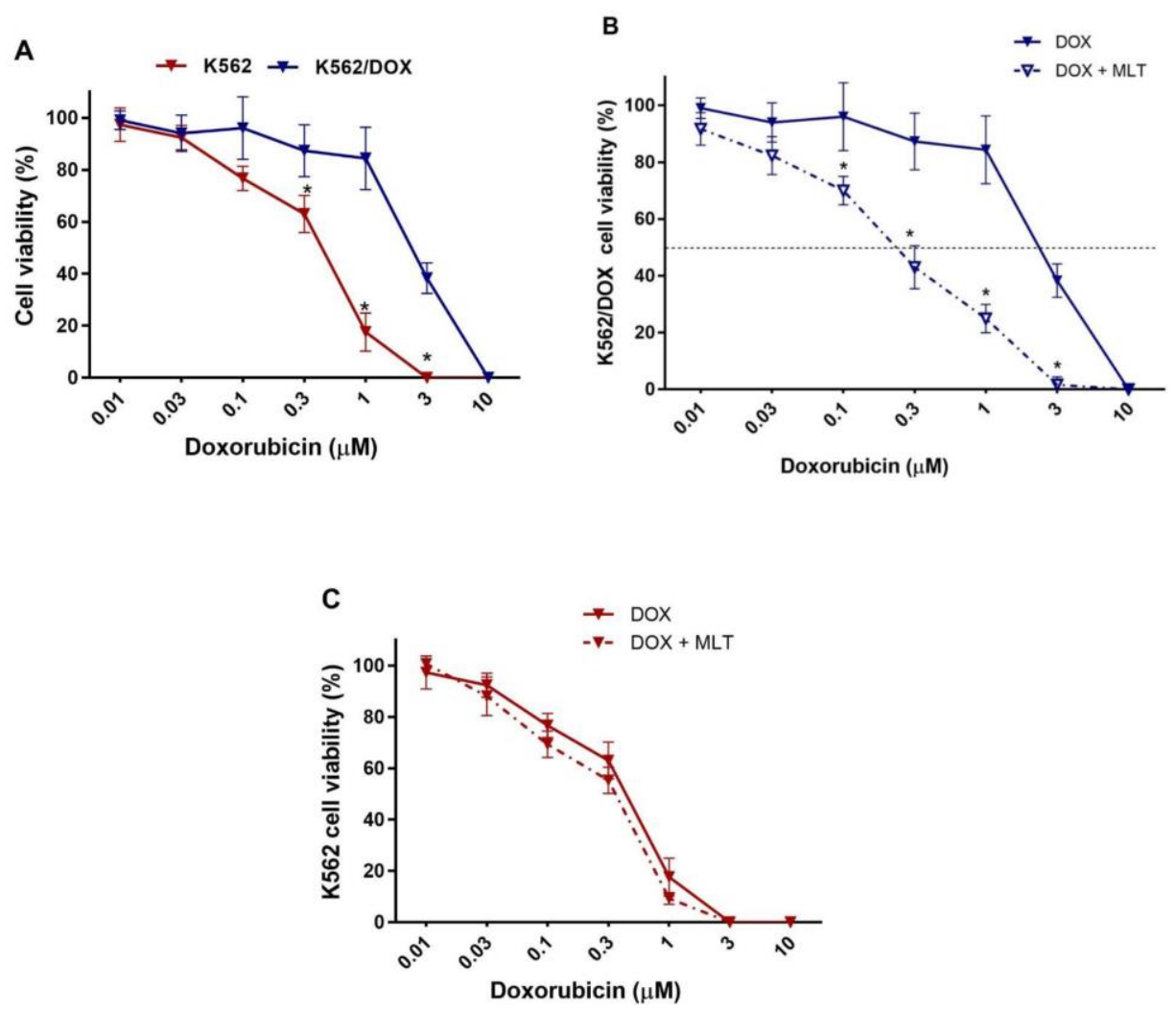

Figure 1. The sensitivity of K562 and K562/DOX cells to DOX in presence and absence of melatonin. (A) Cells were treated with various concentrations of DOX. The viability rates of the cells were measured using an MTT assay after $72 \mathrm{~h}$ of incubation. (B) K562/DOX cells and (C) K562 cells were treated with DOX and DOX plus $300 \mu \mathrm{M}$ of melatonin for $72 \mathrm{~h}$, after which the cell viability was determined. Each point represents the mean \pm SD ( $\mathrm{n}=3$ independent experiments; ${ }^{*} \mathrm{P}<0.05$ vs $\mathrm{K} 562$ /DOX cells).

\subsection{Melatonin treatment decreased P-gp activity}

An intracellular Rho 123 uptake assay was employed to analyze the P-gp activity in overcoming resistance against DOX using melatonin in K562/DOX cells. Treatment with melatonin led to a significant elevation in the intracellular accumulation of Rho 123 after $2 \mathrm{~h}$ in K562/DOX cells (Figure 2A). Parental K562 cells showed no significant effect. Moreover, melatonin also increased the MFI value of K562/DOX cells treated with melatonin following the incubation with Rho 123 in a time-dependent manner $(\mathrm{P}<0.05)$. This effect was similar to the effect of verapamil in K562/DOX cells. No significant change was observed in the parental K562 cells. Therefore, in resistant K562 cells, melatonin combated MDR against DOX by suppressing P-gp activity.

\subsection{Melatonin treatment resulted in the decreased expression of $P$-gp in K562/DOX cells}

The effects of melatonin treatment in resistant 


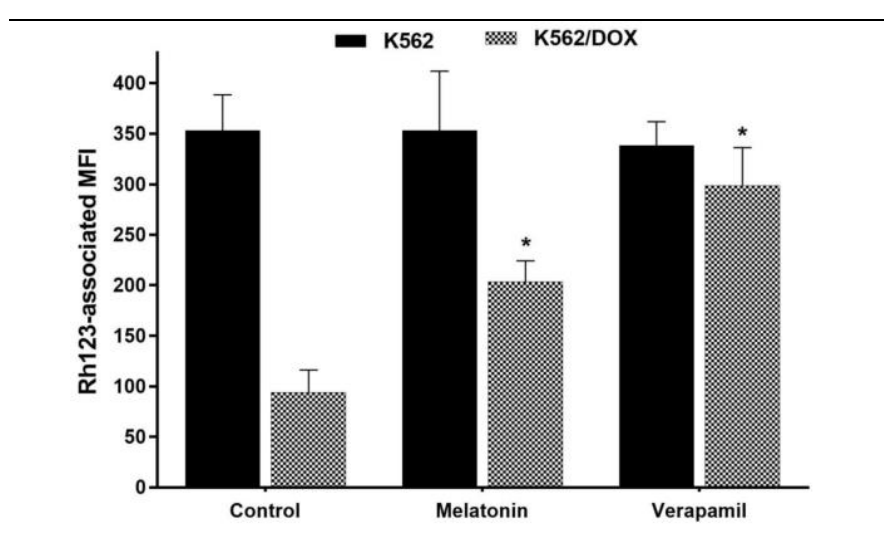

Figure 2. Effect of melatonin on the intracellular accumulation of Rh123. Following culture for $72 \mathrm{~h}$ after melatonin treatment $(300 \mu \mathrm{M})$, the cells were incubated with a medium containing $5 \mu \mathrm{M}$ of Rh123 and $10 \mu \mathrm{M}$ of verapamil for $2 \mathrm{~h}$ and then, Rh123-associated MFI was measured. Each point represents the mean \pm SD from four experiments $\left({ }^{*} \mathrm{P}<0.05\right.$ vs control).

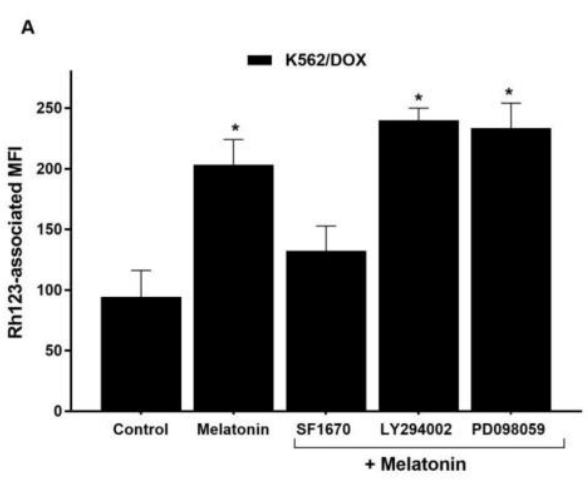

B

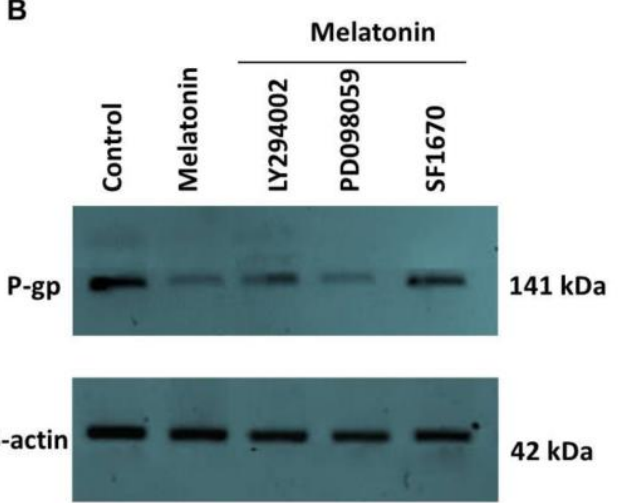

\footnotetext{
Figure 3. Cell viability was determined by MTT reduced activities as described in "Methods". Each value represents the mean \pm SD of three independent experiments. The fold reversal (FR) of MDR was determined by dividing the $\mathrm{IC}_{50}$ of control cells by the $\mathrm{IC}_{50}$ of each experiment. Significantly different from control at aP $<0.05$; No significant difference from melatonin+DOX group.
}

K562 cells were also examined on the protein and mRNA expression levels of P-gp. The mRNA and protein expression levels of P-gp were significantly higher in K562/DOX cells than K562 cells $(\mathrm{P}<0.05)$ (Figures 3A and 3B). Treatment of resistant cells with $300 \mu \mathrm{M}$ melatonin resulted in the significant downregulation of P-gp in K562/DOX cells, which explained the enhanced intracellular concentrations and cytotoxic effects of DOX in resistant K562 cells. Therefore, melatonin can also inhibit the expression of P-gp, which in turn reverses MDR in resistant cells. 
4.4. Melatonin decreased P-gp expression by targeting PTEN

The mRNA and protein-expressing PTEN were measured in K562 and resistant cells, as a tumor suppressor gene, with critical function regulating MDR to evaluate melatonin's impact on PTEN expression levels. Moreover, based on the obtained results, the expression levels of PTEN significantly diminished in resistant cells compared to nonresistant cells. Moreover, the treatment of DOXresistant cells with melatonin led to a significant elevation in mRNA and protein levels of PTEN. Therefore, it is suggested that PTEN may be a direct target of melatonin in reversing MDR

\subsection{PTEN suppression attenuated melatonin mediated anti-MDR effects}

In the next stage, for a better understanding of molecular mechanisms of MDR overcoming in DOX- resistant cells, these cells were treated with LY294002 (PI3K inhibitor), PD098059 (MAPK inhibitor), and SF1670 (PTEN Inhibitor). The combination of either LY294002 or PD098059 with melatonin and DOX significantly reduced resistant cells' IC50 value (Table $1 ; \mathrm{P}<0.05$ ). However, cell treatment with SF1670, as a PTEN inhibitor, increased IC50 value, which in turn abolished the anti-MDR effects of melatonin in resistant cells. Furthermore, evaluating the impact of three inhibitors on the P-gp expression and activity showed that in the presence of SF1670, the P-gp expression and as its activities significantly increased, indicating the attenuation of melatonin mediated positive effects on overcoming MDR in resistant cells through the inhibition of PTEN (Figure 3). Therefore, it is suggested that melatonin reversed MDR in K562/DOX cells by increasing PTEN expression.

\begin{tabular}{lcc}
\hline $\begin{array}{l}\text { Table 1. Effect of melatonin and co-exposure with } \\
\text { cytotoxicity of DOX and reversing MDR in K562/DOX cells }\end{array}$ & \multicolumn{1}{c}{ K562/D0X } \\
\hline \multirow{2}{*}{ Treatment } & $\boldsymbol{I C}_{50}(\boldsymbol{\mu M})$ & $\boldsymbol{F R}$ \\
\hline Control (DOX) & $3.40 \pm 0.317$ & - \\
\hline MLT + DOX & $0.52 \pm 0.11^{\mathrm{a}}$ & 6.53 \\
MLT + LY294002 10 $\boldsymbol{\mu M}+$ DOX & $0.61 \pm 0.18^{\mathrm{a}, \mathrm{b}}$ & 5.57 \\
MLT + PD098059 10 $\boldsymbol{\mu M}+$ DOX & $0.48 \pm 0.08^{\mathrm{a}, \mathrm{b}}$ & 7.08 \\
MLT + SF1670 10 $\boldsymbol{\mu M}+$ DOX & $2.85 \pm 0.31$ & 1.19 \\
\hline
\end{tabular}

A

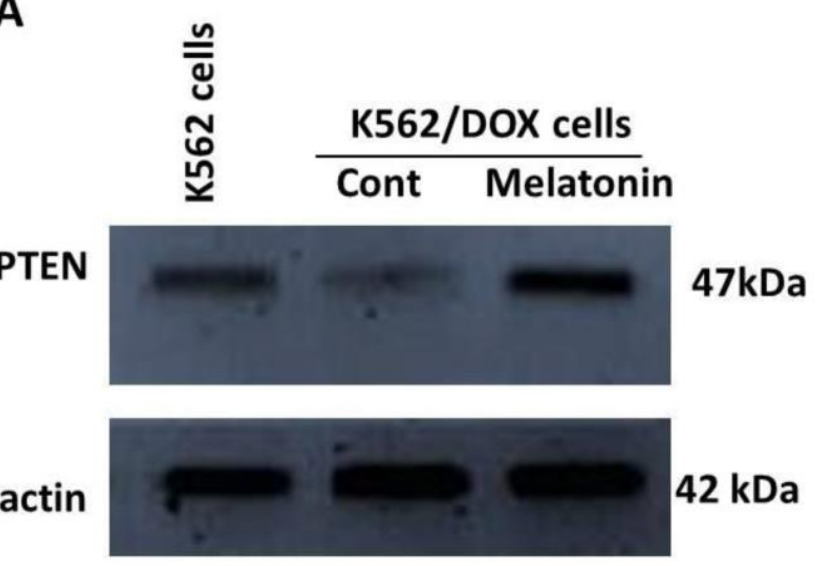

Figure 4.(A) PTEN inhibition abolished melatonin effects on MDR. (A) Effect of LY294002, PD098059, and SF1670 on the melatonin-induced intracellular Rh123 accumulation in K562/DOX cells. Treatment with $10 \mu \mathrm{M}$ of LY294002 or PD098059 increased Rh123-associated MFI. Treatment with $10 \mu \mathrm{M}$ of SF1670 almost abolished the action of the melatonin

\subsection{Melatonin promoted DOX-induced apoptosis in DOX-resistant $K 562$ cells}

Flow cytometry with annexin V /PI staining was applied to evaluate melatonin function in increasing DoX-mediated apoptosis in K562 resistant and parental cells. The obtained results showed that the migration of DOX-resistant K562 cells to apoptotic regions increases in the combined application of melatonin and DOX, compared to DOX alone (Figure 4). 


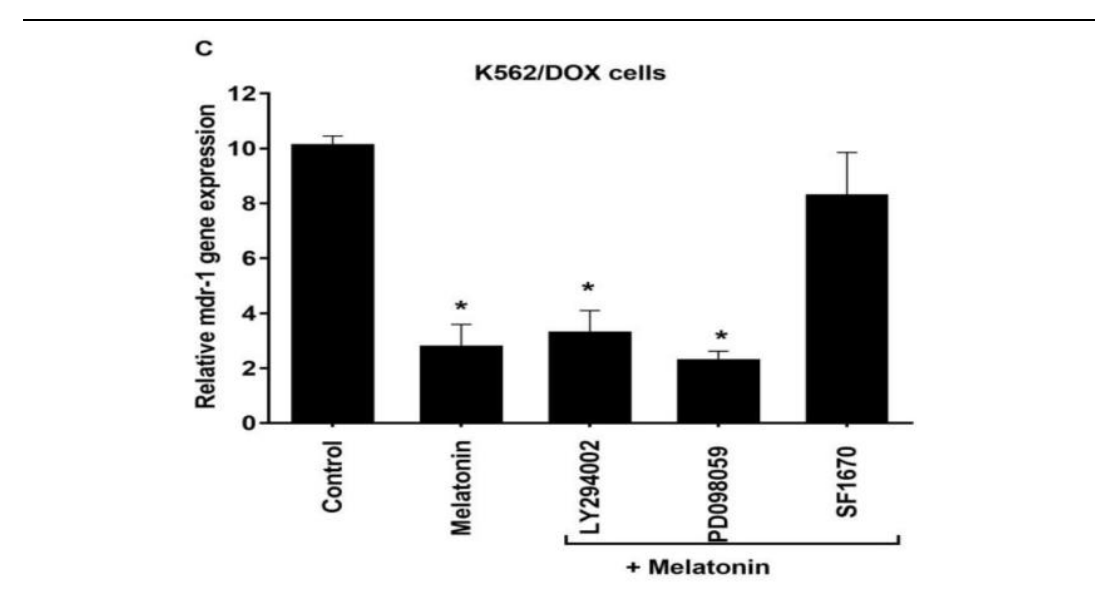

Figure 4.(C) MDR-1 mRNA in K562/DOX cells $72 \mathrm{~h}$ after treatment. SF1670 suppress the effects of melatonin. Data represent mean $\pm \mathrm{SD}\left(\mathrm{n}=3 ;{ }^{*} \mathrm{P}<0.05\right.$ vs untreated k562/DOX cells)

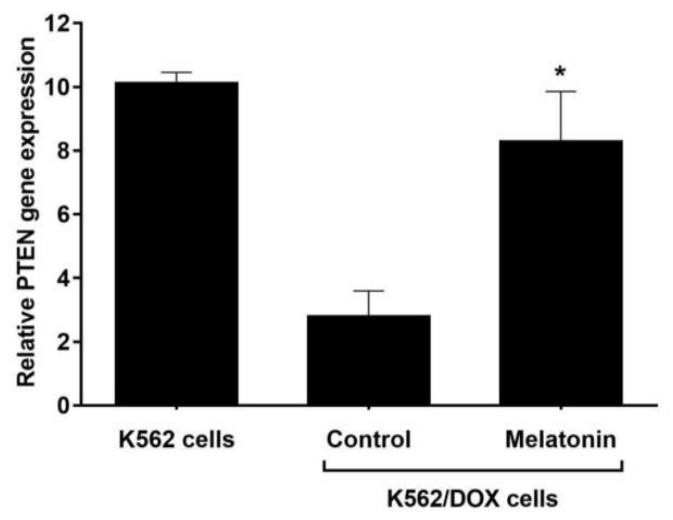

Figure 5. PTEN is a direct target of melatonin. Melatonin upregulated PTEN protein (A) and mRNA levels (B) in K562/DOX cells; the data represent mean \pm SD ( $n=3$, ${ }^{*} \mathrm{P}<0.05$ vs. untreated K562/DOX cells)

B

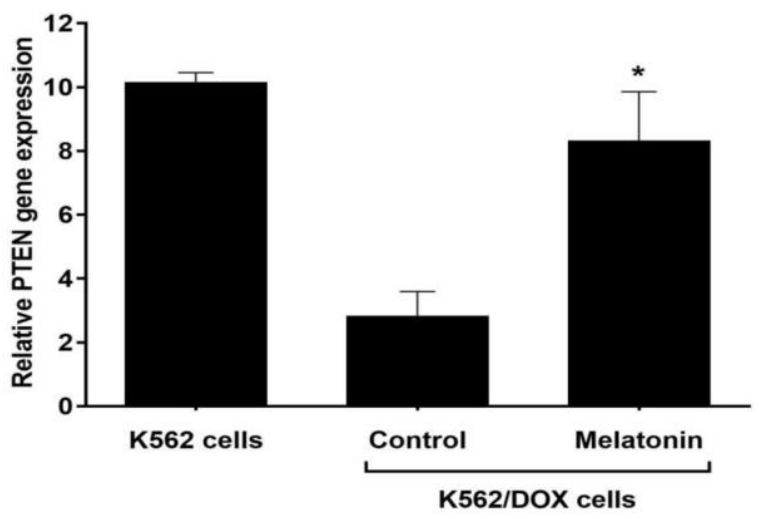

Figure 6. Apoptotic analysis of K562/DOX cells by flow cytometery. Cells were stained with Annexin V-FITC and PI $72 \mathrm{~h}$ after treatment with melatonin $300 \mu \mathrm{M}$ (b), DOX, $0.3 \mu \mathrm{M}$ (c), and melatonin plus DOX, $0.3 \mu \mathrm{M}$ (D).

\section{Discussion}

The study results demonstrated that melatonin effectively increased the cytotoxic impact of DOX in K562/DOX cells through down-regulation of P-gp and up-regulation of PTEN, such that inhibition of PTEN completely abolished the positive effects of melatonin in reversing MDR. The anticancer potential of melatonin has been extensively investigated in recent researches with the promising finding reported in this regard. Notably, an increasing number of recent studies on reversing MDR suggested that the 
combination of melatonin and conventional chemotherapeutics can increase these agents' cytotoxic effects and decrease their adverse side effects. Different mechanisms introduced for antiMDR effects of melatonin in various human malignancies include reducing drug efflux by suppressing the $\mathrm{ABC}$ family, regulating biotransformation and detoxification of drugs, modulating DNA repair machinery, and increasing apoptosis.

One of the most crucial endpoint in fighting MDR includes targeting P-gp, as a well-known member of the ABC family with a critical function in decreasing the intracellular concentrations of drugs and developing MDR. Various studies have demonstrated that melatonin combats MDR through the downregulation of P-gp in cancer cells. Granzotto et al. showed that melatonin caused a significant sensitization to DOX in resistant P388 mouse leukemia. Moreover, DOX intracellular concentrations in resistant P388 leukemia cells were increased by melatonin through the inhibition of P-gp-mediated DOX efflux from the cells (17). In another study performed by Fic et al., the authors found that melatonin intensified the cytotoxicity effect of DOX in the resistant LoVo cells. The combination of melatonin and DOX led to a significant elevation in the percentage of cells expressing P-gp (18). Sun et al. observed that melatonin down-regulated P-gp, which in turn sensitized lymphoma cells to epirubicin that suppressed their growth (19). In line with the results of these studies, findings of the present study showed that the treatment of K562/DOX cells with melatonin decreased DOX IC50 value and increased its cytotoxic effects, as indicated by decreased cellular proliferation and increased apoptotic rate. Melatonin also led to the significant down-regulation of P-gp and decreased K562/DOX cells' activity.

The PTEN is a tumor suppressor gene, which is down-regulated in various human malignancies. The PTEN is downstream of PI3K/Akt signal transduction and has a vital function in cell fate determination (20). The function of PTEN in MDR was widely demonstrated in multiple previous studies (21-24). Xu et al. and Chen et al. showed that PTEN downregulation was crucial for developing resistance against DOX in SGC7901/DOX gastric cancer cells. The activation of PTEN effectively combats $\operatorname{MDR}(25,26)$.

On the other hand, melatonin also increases cancer cells' susceptibility to chemotherapeutic drugs by hindering the expression of key components of the pathway through the inhibition of the PI3K / PTEN pathway, which in turn reverses the induced drug resistance (27-29). The current study results also revealed that the positive effects of melatonin in reversing MDR were mediated through upregulation of PTEN. Inhibition of PTEN by its specific inhibitor abolished the MDR-reversing effects of melatonin.

Regarding the limitation of the present study, one can refer to the lack of in vivo studies and the fact that the results were generated from a cell line. Therefore, a larger-scale study is needed to confirm the current findings. Eventually, organoid models could be helpful in this field as well.

\section{Conclusion}

In the present study, it was demonstrated that the combined application of melatonin and DOX in resistant leukemia cancer cell lines resulted in a potent anticancer effect in cells through circumventing MDR, increasing apoptosis, and targeting PTEN. Therefore, it can be concluded that melatonin might effectively decrease the server-side effects of DOX in cancer patients. Moreover, due to the importance of PTEN activation and apoptotic effects of melatonin in cancer cells in reversing MDR, as demonstrated in this study, there is an urgent need for more in-depth human studies to explain the precise role of melatonin in overcoming MDR and clinical application of melatonin.

\section{Acknowledgments}

The authors would like to thank the Tabriz University of Medical Science, Tabriz, Iran, for supporting this project.

\section{Footnotes}

Authors' contributions: Vahid Rameshknia and Moireh Movahhedi participated in the sampling and data collecting. Maryam Majidinia and Abbas Akhavan wrote the manuscript. Bahman Yousefi designed the study, drafted and revised the manuscript, and interpreted the study data.

Conflicts of Interest: The authors declare that they have no conflict of interest regarding the publication of the present study.

Funding statement: This study was supported by the Tabriz University of Medical Sciences, Tabriz, Iran.

Consent for publication: Not applicable

\section{References}

1. Majidinia M, Mirza-Aghazadeh-Attari M, Rahimi M, Mihanfar A, Karimian A, Safa A, et al. Overcoming multidrug resistance in cancer: recent progress in nanotechnology and new horizons. IUBMB Life. 2020;72(5):855-71. doi: 10.1002/iub.2215. [PubMed: 31913572].

2. Vasan N, Baselga J, Hyman DM. A view on drug resistance in cancer. Nature. 2019;575(7782):299-309. doi: 10.1038/s41586-019-1730-1. [PubMed: 31723286].

3. Zhang J, Gu Y, Chen B. Mechanisms of drug resistance in acute myeloid leukemia. Onco Targets Ther. 2019;12:1937-45. doi: 10.2147/ott.s191621. [PubMed: 30881045].

4. Syed SB, Arya H, Fu IH, Yeh TK, Periyasamy L, Hsieh HP, et al. Targeting P-glycoprotein: Investigation of piperine analogs for overcoming drug resistance in cancer. Sci Rep. 2017;7(1):7972. doi: 10.1038/s41598-017-08062-2. [PubMed: 28801675]. 
5. Chatterjee N, Bivona TG. Polytherapy and targeted cancer drug resistance. Trends Cancer. 2019;5(3):170-82. doi: 10.1016/j.trecan.2019.02.003. [PubMed: 30898264].

6. Yang J, Nie J, Ma X, Wei Y, Peng Y, Wei X. Targeting PI3K in cancer: mechanisms and advances in clinical trials. Mol Cancer. 2019;18(1):26. doi: 10.1186/s12943-019-0954-x. [PubMed: 30782187].

7. Li Y, Guo Y, Feng Z, Bergan R, Li B, Qin Y, et al. Involvement of the PI3K/Akt/Nrf2 signaling pathway in resveratrol-mediated reversal of drug resistance in HL-60/ADR Cells. Nutr Cancer. 2019;71(6):1007-18. doi: 10.1080/01635581.2019.1578387. [PubMed: 31032633].

8. Shi X, Valizadeh A, Mir SM, Asemi Z, Karimian A, Majidina M, et al. miRNA-29a reverses P-glycoprotein-mediated drug resistance and inhibits proliferation via up-regulation of PTEN in colon cancer cells. Eur J Pharmacol. 2020;880:173138. doi: 10.1016/j.ejphar.2020.173138. [PubMed: 32416187].

9. Guerrero-Zotano A, Mayer IA, Arteaga CL. PI3K/AKT/mTOR: role in breast cancer progression, drug resistance, and treatment. Cancer Metastasis Rev. 2016;35(4):515-24. doi: 10.1007/s10555-016-9637-x. [PubMed: 27896521].

10. Chu S, Liu G, Xia P, Chen G, Shi F, Yi T, et al. miR-93 and PTEN: Key regulators of doxorubicin-resistance and EMT in breast cancer. Oncol Rep. 2017;38(4):2401-7. doi: 10.3892/or.2017.5859. [PubMed: 28765915].

11. Najafi M, Salehi E, Farhood B, Nashtaei MS, Hashemi Goradel N, Khanlarkhani N, et al. Adjuvant chemotherapy with melatonin for targeting human cancers: a review. J Cell Physiol. 2019;234(3):2356-72. doi: 10.1002/jcp.27259. [PubMed: 30192001].

12. Xie T, Geng J, Wang Y, Wang L, Huang M, Chen J, et al. FOXM1 evokes 5-fluorouracil resistance in colorectal cancer depending on ABCC10. Oncotarget. 2017;8(5):8574-89. doi: 10.18632/oncotarget.14351. [PubMed: 28051999].

13. Talib WH. Melatonin and cancer hallmarks. Molecules. 2018;23(3):518. doi: 10.3390/molecules23030518. [PubMed: 29495398].

14. Asghari MH, Ghobadi E, Moloudizargari M, Fallah M, Abdollahi M. Does the use of melatonin overcome drug resistance in cancer chemotherapy? Life Sci. 2018;196:143-55. doi: 10.1016/j.lfs.2018.01.024. [PubMed: 29374563].

15. Yousefi B, Azimi A, Majidinia M, Shafiei-Irannejad V, Badalzadeh R, Baradaran B, et al. Balaglitazone reverses Pglycoprotein-mediated multidrug resistance via upregulation of PTEN in a PPAR $\gamma$-dependent manner in leukemia cells. Tumour Biol. 2017;39(10):1010428317716501. doi: 10.1177/1010428317716501. [PubMed: 28978268].

16. Mashayekhi S, Yousefi B, Tohidi E, Darband SG, MirzaAghazadeh-Attari M, Sadighparvar S, et al. Overexpression of tensin homolog deleted on chromosome ten (PTEN) by ciglitazone sensitizes doxorubicin-resistance leukemia cancer cells to treatment. J Cell Biochem. 2019;120(9):15719-29. doi: 10.1002/jcb.28841. [PubMed: 31087712].

17. Cancer Genome Atlas Research Network. Integrated genomic analyses of ovarian carcinoma. Nature. 2011;474(7353):60915. doi: 10.1038/nature10166. [PubMed: 21720365].

18. Fic M, Gomulkiewicz A, Grzegrzolka J, Podhorska-Okolow M, Zabel M, Dziegiel P, et al. The impact of melatonin on colon cancer cells' resistance to doxorubicin in an in vitro study. Int J Mol Sci. 2017;18(7):1396. doi: 10.3390/ijms18071396. [PubMed: 28788434].

19. Liu K, Song J, Yan Y, Zou K, Che Y, Wang B, et al. Melatonin increases the chemosensitivity of diffuse large B-cell lymphoma cells to epirubicin by inhibiting P-glycoprotein expression via the NF-KB pathway. Transl Oncol. 2021; 14(1):100876. doi: 10.1016/j.tranon.2020.100876. [PubMed: 33007707].

20. Di Cristofano A, Pandolfi PP. The multiple roles of PTEN in tumor suppression. Cell. 2000;100(4):387-90. doi: 10.1016/s0092-8674(00)80674-1. [PubMed: 10693755].

21. Tang YA, Chen CH, Sun HS, Cheng CP, Tseng VS, Hsu HS, et al. Global Oct4 target gene analysis reveals novel downstream PTEN and TNC genes required for drug-resistance and metastasis in lung cancer. Nucleic Acids Res. 2015;43(3):1593608. doi: 10.1093/nar/gkv024. [PubMed: 25609695].

22. Retracted: MicroRNA-217 overexpression induces drug resistance and invasion of breast cancer cells by targeting PTEN signaling. Cell Biol Int. 2018;42(10):1455. doi: 10.1002/cbin.10506. [PubMed: 26109338].

23. Steelman LS, Navolanic PM, Sokolosky ML, Taylor JR, Lehmann BD, Chappell WH, et al. Suppression of PTEN function increases breast cancer chemotherapeutic drug resistance while conferring sensitivity to mTOR inhibitors. Oncogene. 2008;27(29):4086-95. doi: 10.1038/onc.2008.49. [PubMed: 18332865].

24. Fang L, Li H, Wang L, Hu J, Jin T, Wang J, et al. MicroRNA-17-5p promotes chemotherapeutic drug resistance and tumour metastasis of colorectal cancer by repressing PTEN expression. Oncotarget. 2014;5(10):2974-87. doi: 10.18632/oncotarget. 1614. [PubMed: 24912422].

25. Xu J, Liu D, Niu H, Zhu G, Xu Y, Ye D, et al. Resveratrol reverses Doxorubicin resistance by inhibiting epithelial-mesenchymal transition (EMT) through modulating PTEN/Akt signaling pathway in gastric cancer. J Exp Clin Cancer Res. 2017;36(1):19. doi: 10.1186/s13046-016-0487-8. [PubMed: 28126034].

26. Chen J, Zhou C, Li J, Xiang X, Zhang L, Deng J, et al. miR - 21- 5p confers doxorubicin resistance in gastric cancer cells by targeting PTEN and TIMP3. Int J Mol Med. 2018;41(4):1855-66. doi: 10.3892/ijmm.2018.3405. [PubMed: 29393355].

27. Fan L, Sun G, Ma T, Zhong F, Lei Y, Li X, et al. Melatonin reverses tunicamycin-induced endoplasmic reticulum stress in human hepatocellular carcinoma cells and improves cytotoxic response to doxorubicin by increasing $\mathrm{CHOP}$ and decreasing survivin. J Pineal Res. 2013;55(2):184-94. doi: 10.1111/jpi.12061. [PubMed: 23711089].

28. Fan L, Sun G, Ma T, Zhong F, Wei W. Melatonin overcomes apoptosis resistance in human hepatocellular carcinoma by targeting survivin and XIAP. J Pineal Res. 2013;55(2):174-83. doi: 10.1111/jpi.12060. [PubMed: 23679681].

29. Yamanishi M, Narazaki H, Asano T. Melatonin overcomes resistance to clofarabine in two leukemic cell lines by increased expression of deoxycytidine kinase. Exp Hematol. 2015;43(3):207-14. doi: 10.1016/j.exphem.2014.11.001. [PubMed: 25461250]. 